\title{
ADAPTIVE APPROACH FOR NON-LINEAR SENSITIVITY ANALYSIS OF REACTION KINETICS
}

\author{
Illia Horenko ${ }^{1 *}$, Sönke Lorenz ${ }^{1}$, Christof Schütte ${ }^{1}$, \\ WilHelm HuisingA ${ }^{1}$ \\ ${ }^{1}$ Freie Universität Berlin, Institut für Mathematik II, \\ Arnimallee 2-6, 14195 Berlin, Germany
}

February 22, 2005

\begin{abstract}
We present a unified approach for linear and non-linear sensitivity analysis for models of reaction kinetics that are stated in terms of systems of ordinary differential equations (ODEs). The approach is based on the reformulation of the ODE problem as a density transport problem described by a Fokker-Planck equation. The resulting multidimensional partial differential equation is herein solved by extending the TRAIL algorithm originally introduced by Horenko and Weiser in the context of molecular dynamics (J. Comp. Chem. 2003, 24, 1921) and discuss it in comparison with Monte Carlo techniques. The extended TRAIL approach is fully adaptive and easily allows to study the influence of non-linear dynamical effects. We illustrate the scheme in application to an enzyme-substrate model problem for sensitivity analysis w.r.t. to initial concentrations and parameter values.
\end{abstract}

keywords: non-linear sensitivity analysis, parameter uncertainty, adaptive particle methods, multidimensional PDEs, Fokker-Planck equation

\section{Introduction}

In computational chemistry and biotechnology, the construction of predictive models has become an essential step in process design and product optimization. Modelling the temporal behavior often leads to systems of ordinary differential equations (ODEs), the numerical solution of which allows to predict the temporal behavior of the system, to optimize parameters, or to study the

*Corresponding author: horenko@math.fu-berlin.de 
sensitivity of the dynamical process w.r.t. its initial values and/or parameters. The latter task, sensitivity analysis, is most of the time either considered in a linearized setting [7], neglecting effects due to the non-linearity of the ODE, or by studying the influence of an initial value or parameter uncertainty on the dynamics via Monte Carlo methods [5, 19, 20]. From a conceptual as well as a practical point of view, this, however, is not satisfactory. Rather, one would like to study the joint influence of all uncertainties based on the fully non-linear dynamics. Mathematically this can be modelled by uncertainty distributions of initial values and/or parameter values that are propagated in time via the underlying ODE.

There are available theoretical and numerical tools for investigating ODEs with initial values and/or parameter uncertainty distributions. One class of approaches is represented by Monte Carlo methods based on a sampling of the parameter space and subsequent solution of the ODE for each of the sampling points (f.e. [19]). While this is the method of choice for problems with many parameters and degrees of freedom in order to avoid the "curse of dimensionality", the questions of numerical accuracy, reliability, and adaptivity still remain partially unclear. A second class of methods is known as the stochastic finite elements (SFEMs) approach $[13,14,15]$. This method represents the overall statistical response of the system by a linear combination of orthogonal basis functions. However, in the available form, this approach cannot be applied to higher dimensional problems with different time and length scales as it is typical for reaction kinetics models.

In this article, we present a theoretical framework and an adaptive numerical approach for systems of ODEs with uncertainty distributions. The approach is based on the reformulation of the problem in terms of a Fokker-Planck partial different equation (PDE). In order to solve the resulting PDE numerically, we adopt and extend the adaptive Gaussian-based particle method TRAIL [11, 12] that has originally been developed in the context of molecular dynamics. The TRAIL approach is based on two ingredients: (i) a linear prediction of the evolving density by means of a particle method using Gaussian ansatz functions, and (ii) a correction of the linear prediction by adaptive approximation of non-linear effects. In this article, we show how the TRAIL algorithm can be extended to allow for non-linear sensitivity analysis and illustrate the performance of the method for the case of a sensitivity analysis of enzyme-substrate reaction kinetics. Moreover, due to the prediction-correction scheme, it is possible to analyze the effects of non-linearity on the overall dynamics by comparing the fully non-linear system to the linearized system. 


\section{Sensitivity analysis reformulated in terms of partial differential equations}

Let us consider the following system of ODEs of the form

$$
\dot{z}=F(z, t), \quad z\left(t_{0}\right)=z_{0},
$$

where $z$ denotes the state vector of the system, $z_{0}$ its initial value at time $t_{0}$, and $F$ the right hand side (RHS) of the ODE. In the context of sensitivity analysis we are interested in the influence of uncertainties in initial values and/or parameters on the dynamical behavior. These uncertainties are modelled in terms of some distribution function $u=u(z, t)$ representing the "spread" of the values. Analyzing the impact of uncertainties corresponds to propagating the initial uncertainty distribution $u\left(\cdot, t_{0}\right)$ under the dynamics of the ODE (1) in time, hence solving for $u(\cdot, t)$ for all $t>t_{0}$. The time propagation of $u$ is described by the Fokker-Planck or Liouville equation [10, 17]

$$
\frac{\partial}{\partial t} u(z, t)=-\sum_{i} \frac{\partial}{\partial z_{i}}(F(z, t) \cdot u(z, t))
$$

with initial density $u=u\left(\cdot, t_{0}\right)$. In the following we will distinguish two cases: (i) $z=x$ for the sensitivity analysis w.r.t. initial conditions (concentrations), and (ii) $z=(x, \theta)$ in the case of the sensitivity analysis w.r.t. initial conditions and parameters (concentrations and rate constants in the language of chemical reaction kinetics).

Sensitivity analysis w.r.t. initial values. Here, we consider the system of ODEs

$$
\dot{x}=f(x, t), \quad x(0)=x_{0},
$$

where $x$ denotes the $d$-dimensional state vector of the system, $x_{0}$ its initial value, and $f$ the RHS. In this case, we consider some density $u=u(x, t)$ depending only on state and time, whose propagation in time is given by the Fokker-Planck equation (2) with $F=f$ :

$$
\frac{\partial}{\partial t} u(x, t)=-\sum_{i=1}^{d}\left(\frac{\partial}{\partial x_{i}} f_{i}(x, t ; \theta) \cdot u(x, t)+f_{i}(x, t ; \theta) \frac{\partial}{\partial x_{i}} u(x, t)\right),
$$

and initial density $u\left(\cdot, t_{0}\right)$.

Sensitivity analysis w.r.t. initial values and parameters. Due to limited experimental precision, in many applications one is interested in the impact of parameter uncertainties. By explicitly considering parameters as state variables, the ODE $(3)$ can be extended to a $(d+p)$ dimensional system of ODEs

$$
\begin{aligned}
\dot{x} & =f(x, \theta, t), & x\left(t_{0}\right) & =x_{0} \\
\dot{\theta} & =0, & \theta\left(t_{0}\right) & =\theta .
\end{aligned}
$$


As a consequence, when analyzing the impact of uncertainty on the dynamical behavior, we have to consider some density $u=u(x, \theta, t)$ depending on space, parameters and time. The corresponding Fokker-Planck equation (2) with $F=$ $(f, 0)$ takes the form:

$$
\begin{aligned}
\frac{\partial}{\partial t} u(x, \theta, t)= & -\sum_{i=1}^{d}\left(\frac{\partial}{\partial x_{i}} f_{i}(x, \theta, t) \cdot u(x, \theta, t)+f_{i}(x, \theta, t) \frac{\partial}{\partial x_{i}} u(x, \theta, t)\right) \\
& -\sum_{i=1}^{p}\left(\frac{\partial}{\partial \theta_{i}} f_{i}(x, \theta, t) \cdot u(x, \theta, t)\right)
\end{aligned}
$$

and initial density $u=u\left(\cdot, \cdot, t_{0}\right)$. The RHS of eqnation (7) consists of the operator acting only on the $x$ coordinate of the overall density $u$ and the whole equation can be understood as a Liouville transport of the density in the phase space of $x$. Due to this fact it is tempting to use some sort of separation ansatz, i.e., $u(x, \theta, t)=u_{1}(x, t) u_{2}(\theta, t)$ and utilize the fact that $u_{2}$ is stationary and so far is time independent. However, as it will be seen in the section 3 and demonstrated in the section 5, the solution of (7) develops non-negligible correlations between the space and parameter coordinates. Such effects cannot be reproduced by the separation ansatz.

\section{Linear sensitivity analysis}

In its simplest version, linear sensitivity analysis models uncertainties related to some state $z_{0}$ in the form of some range $\delta z_{0}[7]$, i.e., we have to consider all possible values $z \in\left[z_{0}-\delta z_{0}, z_{0}+\delta z_{0}\right]$, each being equally likely. Alternatively, a Gaussian or normal distribution centered around $z_{0}$ with variance-covariance matrix related to $\delta z_{0}$ is specified, reflecting the fact that the value $z_{0}$ is thought to be the most likely one, while the neighboring values become less likely with increasing distance.

We here consider the case of uncertainty specified in terms of some Gaussian distribution

$$
g(z, t)=A(t) \cdot \exp \left(\left(z-z_{0}(t)\right)^{T} \mathbf{G}(t)\left(z-z_{0}(t)\right)\right)
$$

with amplitude $A(t)$, center $z_{0}(t)$ and shape matrix $\mathbf{G}(t)$ (equal to the inverse of the covariance matrix). For abbreviation, we define

$$
T(z, t)=\left(z-z_{0}(t)\right)^{T} \mathbf{G}(t)\left(z-z_{0}(t)\right)
$$

and obtain $g(z, t)=A(t) \exp (T(z, t))$. In the setting of linear sensitivity analysis, we are interested in the solution of the Fokker-Planck PDE (2) w.r.t. the linearized RHS $D F\left(z_{0}(t)\right)$ and density $u=g$. As it will turn out, this can easily be achieved by simply inserting the Gaussian function (8) into the Fokker-Planck equation and propagating the derived ODEs for amplitude, center and shape 
matrix of the Gaussian. This way, the solution of PDE (4) corresponding to the linearized ODE $\dot{y}=D F\left(z_{0}(t)\right) y$ drastically simplifies. Computing the spatial and temporal partial derivatives for $g$, inserting into (4) and dividing by $\exp (T(z, t))$ yields

$$
\frac{\partial}{\partial t} A(t)+A(t) \frac{\partial}{\partial t} T(z, t)=-A(t) \sum_{i}\left(\frac{\partial}{\partial z_{i}} F_{i}(z, t)+F_{i}(z, t) \frac{\partial}{\partial z_{i}} T(z, t)\right) .
$$

As mentioned above, we linearize the underlying dynamics, i.e., we linearize the RHS in (1) around the center $z_{0}(t)$ of the Gaussian,

$$
F(z, t) \approx F\left(z_{0}(t), t\right)+\mathbf{J}_{z}\left(z-z_{0}(t)\right),
$$

with $\mathbf{J}_{z}=D F(z)$ being the Jacobian of $F$ at $z_{0}$. Inserting into (10) yields terms that are constant, linear, and quadratic in $\left(z-z_{0}(t)\right)$. Separating the different contributions results in

$$
\begin{aligned}
\dot{A} & =-A \cdot \operatorname{trace}\left(\mathbf{J}_{z}\right) \\
\dot{z_{0}} & =F\left(z_{0}, t\right) \\
\dot{\mathbf{G}}(t) & =-\mathbf{J}_{z} \mathbf{G}(t)-\mathbf{G}(t) \mathbf{J}_{z}^{T} .
\end{aligned}
$$

Whenever the shape matrix is symmetric for some time $t_{0}$, i.e., $\mathbf{G}\left(t_{0}\right)=\mathbf{G}\left(t_{0}\right)^{T}$, eq. (13) yields $\dot{\mathbf{G}}\left(t_{0}\right)=\dot{\mathbf{G}}\left(t_{0}\right)^{T}$. As a consequence, symmetry is preserved for all times $t \geq t_{0}$, such that $\mathbf{G}$ indeed defines a shape matrix of a Gaussian. Looking at the analytical solution of (13), the analytical solution

$$
\mathbf{G}(t)=\exp \left(-\mathbf{J}_{z} t\right) \cdot \mathbf{G}(0) \cdot \exp \left(-\mathbf{J}_{z} t\right)^{T} .
$$

one easily see that $\mathbf{G}$ remains positive semi-definite, too. Therefore, our solution for $\mathbf{G}$ fulfills the requirements of a shape matrix.

Summarizing, instead of solving the Fokker-Planck PDE (2) for the linearized ODE $\dot{y}=\mathbf{J}_{z} y$ and with $u=g$ to determine $g(\cdot, t)$, we simply have to solve the system of ODEs (11)-(13) that describe the time evolution of the corresponding parameters. Then, $g(\cdot, t)$ is obtained via relation (8).

Sensitivity analysis w.r.t. initial values. Simply set $z=x, z_{0}=x_{0}, F=f$ and $\mathbf{J}_{z}=D f\left(x_{0}\right)$ to obtain the required evolution equations for the parameters of the Gaussian distribution.

Sensitivity analysis w.r.t. initial values and parameters. Set $z=(x, \theta)$, $z_{0}=\left(x_{0}, \theta_{0}\right), F=(f, 0)$ and $\mathbf{J}_{z}=D(f, 0)\left(x_{0}, \theta_{0}\right)$ in order to obtain the required evolution equations for the parameters of the Gaussian distribution. Due to the special structure of the RHS, the evolution equation for the shape matrix can be further simplified: The general shape matrix of the Gaussian density $g=g(x, \theta, t)$ in state and parameter space has the structure

$$
\mathbf{G}=\left(\begin{array}{cc}
\mathbf{G}_{x} & \mathbf{G}_{m} \\
\mathbf{G}_{m}^{T} & \mathbf{G}_{\theta}
\end{array}\right)
$$


The matrices $\mathbf{G}_{x}$ and $\mathbf{G}_{\theta}$ describe the statistic interdependencies within the state and parameter space, respectively, while the matrix $\mathbf{G}_{m}$ describes the ones between the state and parameter space. Denote by $\mathbf{J}_{x}$ and $\mathbf{J}_{\theta}$ the Jacobians of $f$ in (5) w.r.t. the spatial coordinates $x$ and the parameters $\theta$, respectively. Then, the Jacobian $\mathbf{J}_{(x, \theta)}$ of the RHS of (5) and (6) has the special form

$$
\mathbf{J}_{(x, \theta)}=\left(\begin{array}{cc}
\mathbf{J}_{x} & \mathbf{J}_{\theta} \\
0 & 0
\end{array}\right)
$$

This allows to establish evolution equations for the sub-matrices of $\mathbf{G}$ :

$$
\begin{aligned}
\dot{\mathbf{G}}_{x} & =-\mathbf{J}_{x} \mathbf{G}_{x}-\mathbf{J}_{\theta} \mathbf{G}_{m}^{T}-\left(\mathbf{J}_{x} \mathbf{G}_{x}+\mathbf{J}_{\theta} \mathbf{G}_{m}^{T}\right)^{T} \\
\dot{\mathbf{G}}_{m} & =-\mathbf{J}_{x} \mathbf{G}_{m}-\mathbf{J}_{\theta} \mathbf{G}_{\theta} \\
\dot{\mathbf{G}}_{\theta} & =0
\end{aligned}
$$

The analytical solution for the entire shape matrix is then given by

$$
\mathbf{G}(t)=\left.\exp \left\{-\left(\begin{array}{cc}
\mathbf{J}_{x} & \mathbf{J}_{\theta} \\
0 & 0
\end{array}\right) t\right\}\left(\begin{array}{cc}
\mathbf{G}_{x} & 0 \\
0 & \mathbf{G}_{\theta}
\end{array}\right)\right|_{t=0} \exp \left\{-\left(\begin{array}{cc}
\mathbf{J}_{x} & \mathbf{J}_{\theta} \\
0 & 0
\end{array}\right) t\right\}^{T} .
$$

Extension to non-Gaussian densities. Due to linearity of the FokkerPlanck PDE, the linear sensitivity analysis can easily be extended to the case of some initial density $u=u\left(z, t_{0}\right)$ being represented as a sum of Gaussian distributions $g_{j}$, i.e.,

$$
u\left(z, t_{0}\right)=\sum_{j=1}^{M} g_{j}\left(z, t_{0}\right)
$$

The above derivation allows for the computation of the density at time $t$

$$
u(z, t)=\sum_{j=1}^{M} A_{j}(t) \exp \left(T_{j}(z, t)\right)
$$

However, still $u(\cdot, t)$ is based on a linearized dynamics only and thus will only be an approximation of the solution of (4) based on the fully non-linear system. This will be accounted for in the non-linear sensitivity analysis.

\section{Non-linear sensitivity analysis}

For the density propagation of Liouville type problems, Horenko and Weiser developed a multidimensional, fully adaptive particle method to describe the propagation of distributions in non-linear dynamical systems, called TRAIL (Trapezoid Rule for Adaptive Integration of Liouville dynamics) [11, 12]. The adaptive discretization scheme is based on the Rothe method $[6,8,18]$. The key 
idea is to approximate the distribution $u$ as a superposition of Gaussian distributions that are adapted in time corresponding to the non-linear dynamics. In each time step $t \rightarrow t+\Delta \tau$, the scheme comprises two steps: In the first step, the Fokker-Planck PDE problem for the corresponding linearized ODE is solved. This transport along the characteristics of the linearized dynamics results in an approximation - a so-called prediction - of the solution. In the second step, the prediction is then refined in order to account for non-linear effects and to meet the accuracy requirements. This step is called correction.

The propagation of the density w.r.t. the linearized ODE is one of the key ingredients of the TRAIL scheme. As it turns out, for the type of ODE considered herein, this prediction is identical to the solution of the linear sensitivity analysis presented above. Thus, the TRAIL approach, in one of its key steps, is based on linear sensitivity analysis. However, the corresponding solution, here denoted by $u_{\text {pred }}$, is in the subsequent correction step further refined to account for non-linear effects. This is done in an adaptive manner such that the final approximation $u_{\text {corr }}$ satisfies the used-prescribed accuracy requirement

$$
\left\|u(\cdot, t+\tau)-u_{\mathrm{corr}}(\cdot, t+\tau)\right\|<\mathrm{tol}
$$

for some suitable norm $\|\cdot\|$. We now sketch the algorithmic flow of the extended TRAIL approach; for details we refer to [11, 12]. Assume, we are given some initial uncertainty density $u=u\left(z, t_{0}\right)$. In a first step, $u\left(\cdot, t_{0}\right)$ is approximated by a finite sum of Gaussian distributions

$$
u\left(z, t_{0}\right)=\sum_{j=1}^{M} A_{j}\left(t_{0}\right) \exp \left(T_{j}\left(z, t_{0}\right)\right)+\epsilon_{t_{0}}
$$

such that $\left\|\epsilon_{t_{0}}\right\|<$ tol. In the second step, the Gaussian distributions are propagated for some adaptively chosen time step $\tau>0$ resulting in the prediction

$$
u_{\text {pred }}\left(z, t_{0}+\tau\right)=\sum_{j=1}^{M} A_{j}\left(t_{0}+\tau\right) \exp \left(T_{j}\left(z, t_{0}+\tau\right)\right) .
$$

In a third step, this prediction is then refined. Two types of refinements are considered: adaption of the amplitudes $A_{j}$ and adaption of the number $M$ of Gaussians. For the adaption of the amplitude, the optimization problem

$$
\left\|\bar{u}(\cdot, t+\tau)-u_{\mathrm{corr}}(\cdot, t+\tau)\right\|=\min _{\left(A_{j}\right)_{j=1, \ldots, M}},
$$

is solved. Here the distribution $\bar{u}$ is defined exploiting the implicit Trapezoid rule for semi-discretization in time

$$
\left(1-\frac{\tau}{2} \mathcal{L}\right) \bar{u}(\cdot, t+\tau)=\left(1+\frac{\tau}{2} \mathcal{L}\right) u(\cdot, t)
$$

with infinitesimal generator $\mathcal{L} u=-\operatorname{div}(F \cdot u)$ corresponding to the FokkerPlanck equation (2). Since the centers $z(t+\tau)$ and the shape matrices $G(t+$ 
$\tau)$ are considered to be fixed, the problem (21) is only optimizing over the amplitudes $A_{t}(t+\tau)$. This optimization problem can be reformulated as a least squares problem, whose solution require the use of the QR-algorithm [7]. If the user-prescribed accuracy requirement in (21) cannot be fulfilled, the number of Gaussian ansatz functions is increased (spawn), and the optimization problem is redone. Moreover, it might also be necessary to decrease (prune) the number of Gaussian, whenever the distribution starts to show a simpler structure or, for reasons of numerical stability, whenever two Gaussians are too close together. In the spawning case, the local error estimator of the implicit Trapezoid rule is employed to spot the particles in the vicinity of which new Gaussians are to be created. Compared to the purely heuristical spawning techniques presented e.g. in $[2,4,3]$, the spawning within trail in controlled by the global tolerance. In the pruning case, an analysis of the problem's sub-condition number is used to spot the particles that ought to be removed. Both of these steps can be done adaptively and simultaneously with the solution of (21) in terms of the QR-decomposition. Details may again be found in [11]. In the final step, the estimation error resulting from the Trapezoid rule is also employed to compute the next time step $\tau$; this is done by standard methods of time step control [7].

\section{Numerical examples}

We now apply and compare the extended TRAIL algorithm with other numerical methods for some simple non-linear model of reaction kinetics. In 1913, Michaelis and Menten introduced a model for the conversion of a substrate $S$ to a product $P$ catalyzed by some enzyme $E[1,9,16]$. The reaction is assumed to take part in two steps. First, the substrate $S$ and enzyme $E$ form an enzymesubstrate complex $E S$, which is subsequently irreversibly transformed into the product $P$.

$$
E+S \underset{k_{1}^{-}}{\stackrel{k_{1}^{+}}{\rightleftarrows}} E S \stackrel{k_{2}^{+}}{\longrightarrow} P+E
$$

$k_{1}^{-}, k_{1}^{+}$and $k_{2}^{+}$denote here the corresponding reaction rates. The total concentration of the enzyme $[E]_{T}$ and the substrate $[S]_{T}$ satisfy the conservation conditions

$$
\begin{aligned}
{[E]_{T} } & =[E]+[E S] \\
{[S]_{T} } & =[S]+[E S]+[P] .
\end{aligned}
$$

Since the reaction involving $k_{2}^{+}$is irreversible, we may completely characterize the catalytic reaction by the system of two ODEs

$$
\begin{aligned}
\frac{\mathrm{d}}{\mathrm{d} t}[S] & =-k_{1}^{+}[E]_{T}[S]+\left(k_{1}^{-}+k_{1}^{+}[S]\right)[E S] \\
\frac{\mathrm{d}}{\mathrm{d} t}[E S] & =k_{1}^{+}[E]_{T}[S]-\left(k_{1}^{-}+k_{2}^{+}+k_{1}^{+}[S]\right)[E S] .
\end{aligned}
$$


The above ODEs can further be simplified, if $[E]_{T} \ll[S]$, resulting in the wellknown Michaelis-Menten or Monod approximation [9]. However, in the following we will analyze the full model.

\subsection{Sensitivity analysis w.r.t. initial values}

The above model is now used to illustrate and compare the extended TRAIL approach with alternative methods. In the first example, we analyze the effect of initial value uncertainties; hence we regard the initial concentrations $[S](0)$ and $[E S](0)$ as being distribution functions. In order to keep the example simple, we assume that the uncertainties are normally distributed according to

$$
[S](0) \sim \mathcal{N}\left(0.7, \frac{1}{\sqrt{10}}\right),[E S](0) \sim \mathcal{N}\left(1, \frac{1}{\sqrt{10}}\right)
$$

and specify the reaction constants as $k_{1}^{+}=1, k_{1}^{-}=1, k_{2}^{+}=1,[E]_{T}=1$. Four numerical methods have been applied to analyze the sensitivity of the system of ODEs (26) and (27) w.r.t. the initial value uncertainty:

1. Finite element method (FEM) with a uniform $200 \times 200$ grid

2. Monte Carlo sampling (MC) of the initial value distributions and subsequent trajectory propagation

3. Extended TRAIL approach

4. Linear sensitivity analysis

Due to the low-dimensional (2d) character of the problem, the FEM can be used to numerically compute a reference solutions with high accuracy. Figure 1 shows the FEM reference solution in comparison to the solutions obtained by the remaining three approaches. We observe that the extended TRAIL approach (with tol $=0.01$ yielding a representation with only 20 Gaussian ansatz functions) as well as the MC method (with 20.000 sampling points) produce very good results. The linear sensitivity analysis yields a misleading result; this clearly indicates that already for this simple model system, non-linear effects play an important role.

\subsection{Sensitivity analysis w.r.t. initial values and parame- ters}

We now illustrate the sensitivity analysis for uncertain initial values and parameters. The initial values for $[S]$ and $[E S]$ as well as the four parameters $k_{1}^{-}, k_{1}^{+}$, 

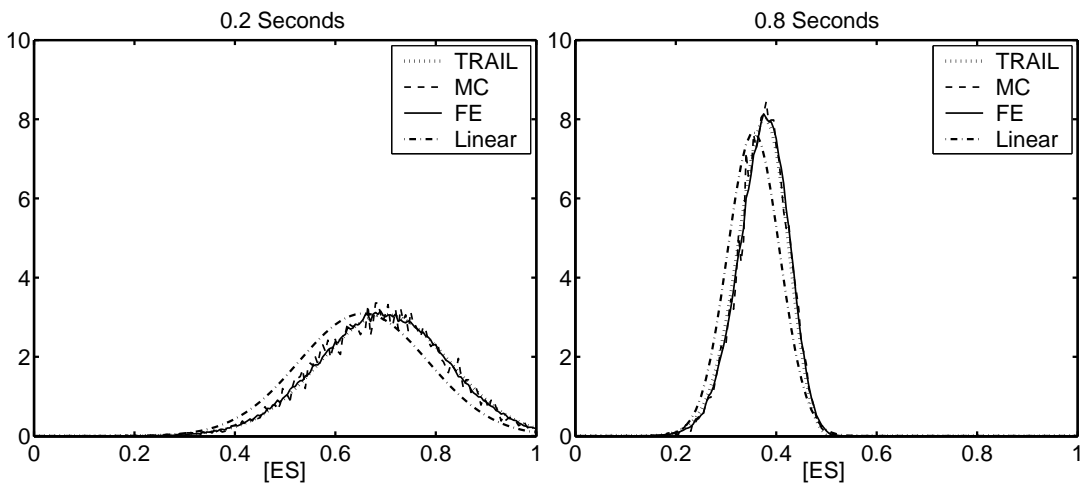

Figure 1: Propagated densities at times $t=0.2$ and $t=0.8$ for the model system (26)-(27) with uncertainty in initial conditions given by (28). Comparison of solutions generated by means of the four schemes introduced in the text. The solutions of FEM, extended TRAIL and MC are very close together, while the linearized propagation results in wrong densities. Note that in contrast to FEM or extended TRAIL, the solution obtained with MC shows some considerable roughness.

$k_{2}^{+}$and $[E]_{T}$ are assumed to be normally distributed according to

$$
\begin{aligned}
& {[S](0) \sim \mathcal{N}\left(8, \frac{1}{\sqrt{40}}\right),[E S](0) \sim \mathcal{N}\left(2, \frac{1}{\sqrt{40}}\right),} \\
& k_{1}^{-} \sim \mathcal{N}\left(0.5, \frac{1}{\sqrt{20}}\right), k_{1}^{+} \sim \mathcal{N}\left(0.3, \frac{1}{\sqrt{20}}\right), \\
& k_{2}^{+} \sim \mathcal{N}\left(2, \frac{1}{\sqrt{20}}\right) \text { and }[E]_{T} \sim \mathcal{N}\left(2, \frac{1}{\sqrt{20}}\right) .
\end{aligned}
$$

By considering the initial concentrations and the parameters as uncertain, the PDE problem now becomes six dimensional. Due to the immense computational effort, the finite element method has to be rejected as a suitable method, such that only the Monte Carlo simulation remains for comparison. Figure 2 demonstrates the convergence of the MC method with increasing number of sampling points (10.000 vs. 100.000) and the extended TRAIL approach. It can be seen that the quality of the MC approximation deteriorates with time (increasing roughness) which is due to the non-conservative structure of the underlying dynamics. In Figure 3 we compare the results of the extended TRAIL approach (with tol $=0.06$ ) to a high precision Monte Carlo simulation (650.000 trajectories). The results of the extended TRAIL approach almost perfectly correspond to the Monte Carlo results. When decreasing the user-prescribed tolerance to tol $=0.03$, the approximation quality increases considerably, as can be seen. Figure 4 illustrates how the time step $\tau$ and the number $M$ of Gaussians used to approximate the distribution change during the course of the propagation 
for the two chosen tolerances. Particularly one observes that (as always) higher accuracy requires higher computational effort. Generally speaking, the computation time difference between TRAIL and Monte Carlo for acceptable tolerance cover $1-2$ order of magnitude. 

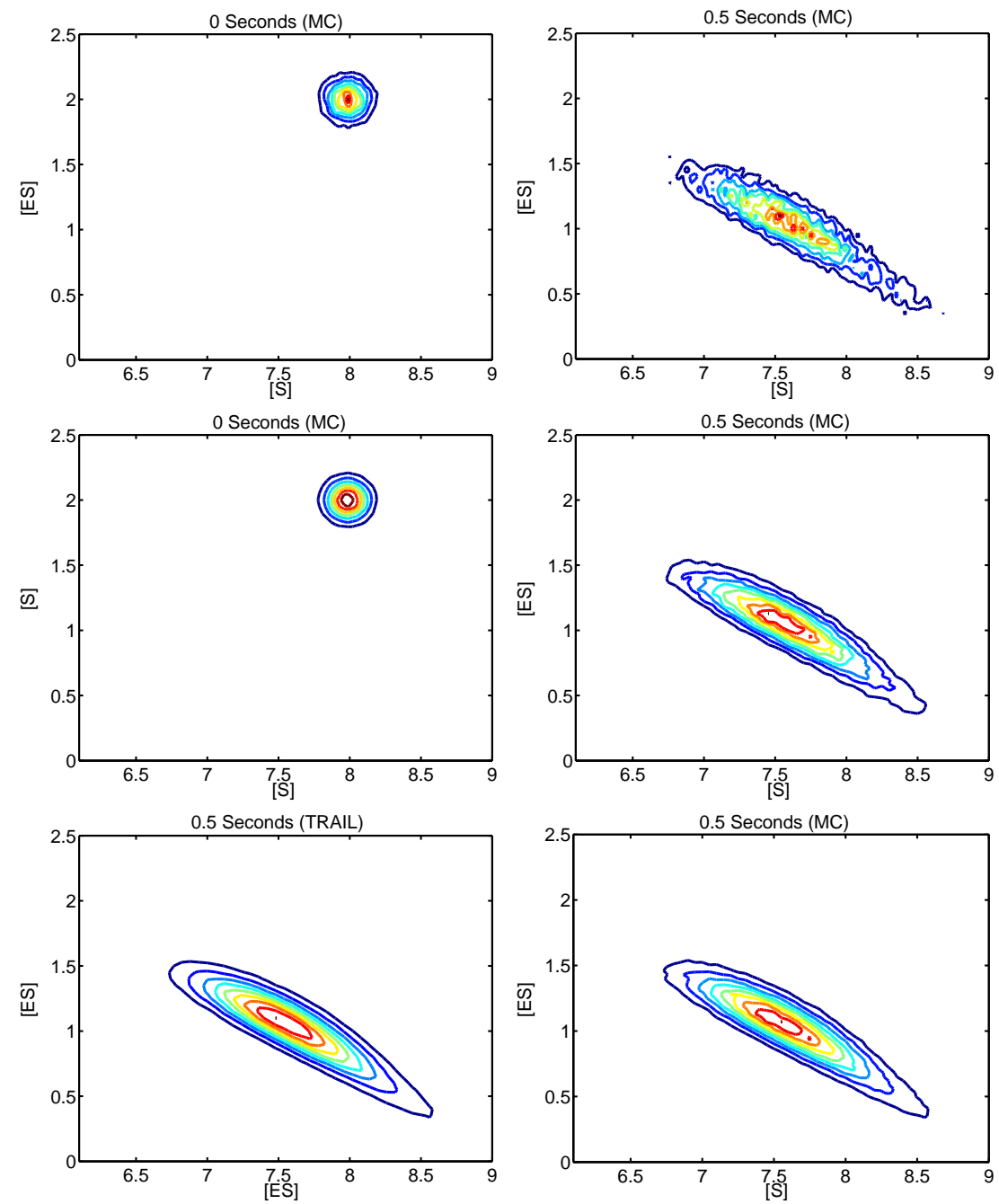

Figure 2: MC solutions for instances $t=0.0 \mathrm{~s}$ and $0.5 \mathrm{~s}$ and different numbers of sampling trajectories: 10.000 (top) 100.000 (middle) and 300.000 (bottom, right). At the bottom left, the TRAIL solution for tol $=0.03$ is shown. 

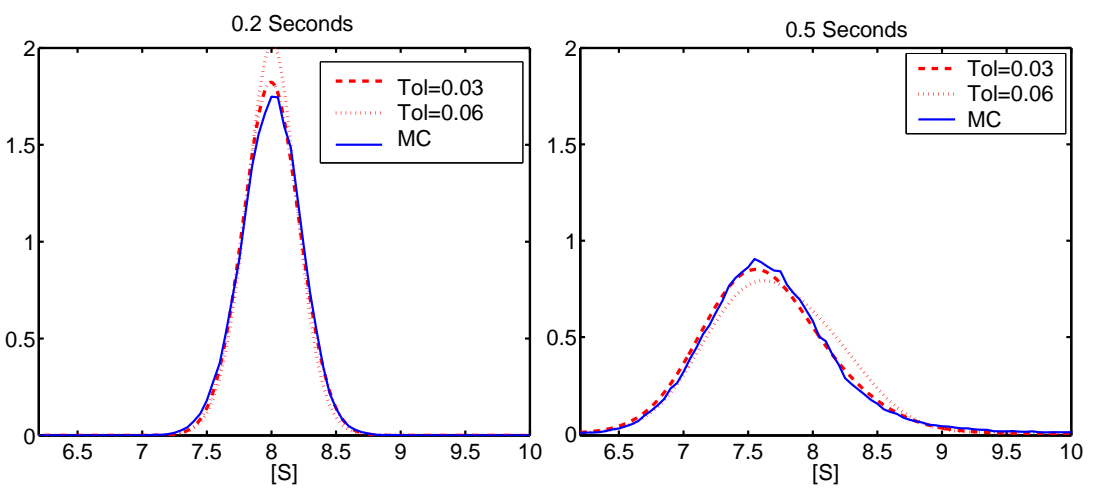

Figure 3: Propagated densities at $t=0.2$ and 0.5 . Note that the distribution develops asymmetry and that the approximation quality improves when the user-prescribed tolerance tol is decreased ( $\mathrm{tol}=0.06 \mathrm{vs}$. tol $=0.03$ ).
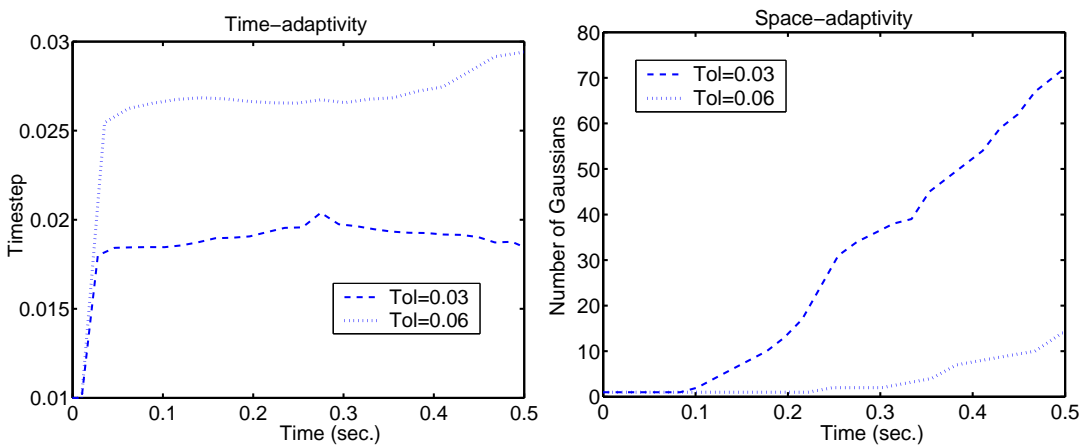

Figure 4: Adaptivity in time and space. Left: Behavior of the time step $\tau$ versus time for two different tolerances. Right: Number of Gaussian ansatz functions generated within the adaptive control versus time for two different tolerances. 


\section{Conclusion}

In this article we analyze the problem of non-linear sensitivity analysis for models of reaction kinetics. We show how the problem can be reformulated in the context of Fokker-Planck equations, i.e., PDEs that describes the evolution of densities w.r.t. the dynamics specified by the underlying ODE. This allows us to apply different methods for solving such time dependent PDEs, like FEM, or MC methods.

As a promising alternative approach, we derive an extension of the TRAIL approach that allows to reliably tackle problems of non-linear sensitivity analysis. The extended TRAIL scheme is implemented as a fully adaptive particle method, where the number of particles is controlled by a user-prescribed tolerance. It is worth noticing that the numerical effort scales with the number of particles used and is independent of the problem's dimension. We show that the linear and non-linear sensitivity analysis can be considered in a unified way within the framework of TRAIL.

We demonstrate that the FEM, MC and the extended TRAIL approach yield comparable results in application to the sensitivity analysis for initial concentrations of an enzyme-substrate model problem. When also taking the uncertainty of parameters into account, only MC and TRAIL obtain results with reasonable computational effort. In comparison, these two approaches are conceptually quite different: The $\mathrm{MC}$ approach yields as a result an ensemble of sampling points. In contrast to molecular dynamics, where the ODE is of Hamiltonian structure and we thus get conservation of the phase volume and $u(x(t), t)=u\left(x\left(t_{0}\right), t_{0}\right)$, in the field of reaction kinetics, this property does in general not hold. Thus, a single sampling point is of limited use and only via the formation of expectations the information contained in the entire ensemble is accessible. For example, in order to determine the value $u(x, t)$ at a specific point $x$, we have to introduce some discretization box $B(x)$ containing $x$ and then compute the ensemble average over $B(x)$. The extended TRAIL algorithm yields as a result a continuous approximation of the density in time. As a consequence, point information like $u(x, t)$ are easily accessible. However, problems involving distribution functions with large degree of spatio-temporal details tend to increase the number of needed Gaussian distributions significantly and consequently increase the numerical effort. Alternative approaches are currently under investigation. The scheme underlying the TRAIL approach is not restricted to Gaussian particles as ansatz functions and can be based on any set of distribution functions, assuming the existence of a reliable analogy to the linear predictor (c.f. (11), (12) and (13)) for this functions.

Acknowledgement. IH acknowledges support by SfB 450, SL acknowledges sponsering by BASF AG, Ludwigshafen, Germany, CS and WH acknowledges support by DFG research center MATHEON. 


\section{References}

[1] J. E. Bailey and D. F. Ollis. Biochemical engineering Fundamentals. McGrawHill, Inc., 2nd edition, 1986.

[2] M. Ben-Nun and T. J. Martinez. Nonadiabatic molecular dynamics: Validation of the multiple spawning method for a multidimensional problem. Journal of Chemical Physics, 108(17):7244-7257, 1998.

[3] M. Ben-Nun and T. J. Martinez. Ab initio quantum molecular dynamics. Adv. Chem. Phys., 121:439-512, 2002.

[4] M. Ben-Nun, J. Quenneville, and T. J. Martinez. Ab initio multiple spawning: Photochemistry from first principles quantum molecular dynamics. Journal of Physical Chemisty A, 104(22):5161-5175, 2000.

[5] P. Bernillon and F. Bois. Statistical issues in toxicokinetic modelling: a Bayesian perspective. Environmental Health Perspectives, 108(Suppl. 5):883-893, 2000.

[6] F. A. Bornemann. An adaptive multilevel approach to parabolic equations: I. general theory and $1 \mathrm{~d}$ implementation. Impact of computing in science and engineering, 2:279-317, 1990.

[7] P. Deuflhard and F. Bornemann. Scientific Computing with Ordinary Differential Equiations. Texts in Applied Mathematics. Springer, 2002.

[8] P. Deuflhard, J. Lang, and U. Nowak. Recent progress in dynamical process simulation. In H. Neunzert, editor, Progress in Industrial Mathematics, pages 122-137. 8th Conference of the European Consortium for Mathematics in Industry (ECMI 94), Wiley \& Teubner Publishers, 1996.

[9] C. P. Fall, E. S. Marland, J. M. Wagner, and J. J. Tyson. Computational Cell Biology, volume 20 of Interdisciplinary Applied Mathematics. Springer Verlag New York Inc., 2002.

[10] C. W. Gardiner. Handbook of Stochastic Methods. For Physics, Chemistry and the Natural Sciences, volume 13 of Springer Series in Synergetics. Springer Verlag Berlin Heidelberg, second edition, 2002.

[11] I. Horenko and M. Weiser. Adaptive integration of molecular dynamics. Journal of Computational Chemistry, 24(15):1921-1929, 2003.

[12] I. Horenko, M. Weiser, B. Schmidt, and C. Schütte. Fully adaptive propgation of the quantum-classical liouville equation. Journal of chemical physics, 120(19):8913-8923, 2004.

[13] A. Keese. A review of recent developments in the numerical solution of stochastic partial differential equations (stochastic finite elements). Informationbericht 2003-6, Department of Computer Science, Technical University Braunschweig, Brunswick, Germany, Institute of Scientific Computing, 
Technische Universität Braunschweig, Hans-Sommer-Straße 65, D-38106 Braunschweig, October 2002.

[14] M. Kleiber and T. D. Hien. The stochastic finite element method, Basic perturbation technique and computer implementation. J. Wiley and Sons, 1992.

[15] H. G. Matthies and M. Meyer. Nonlinear galerkin methods for the model reduction of nonlinear dynamical systems. Informationsberich 20023, Department of Computer Science, Technical University Braunschweig, Brunswick, Germany, March 2002. submitted to special issue of Computers and Structures, Revised and extended version of a contribution to the EUROMECH Colloquium, 427, ENS Cachan France, September 2001.

[16] L. Michaelis and M. L. Menten. Die Kinetik der Invertinwirking. Biochemische Zeitschrift, 49:333-369, 1913.

[17] H. Risken. The Fokker-Planck Equation. Methods of Solution and Applications, volume 18 of Springer Series in Synergetics. Springer Verlag, second edition, 1996.

[18] E. Rothe. Zweidimensionale parabolische Randwertaufgabe als Grenzfall eindimensionaler Randwertaufgaben. Mathematische Annalen, 102:650$670,1930$.

[19] D. Talay. Probabilistic numerical methods for partial differential equations: elements of analysis. In D. Talay and L. Tubaro, editors, Probabilistic Models for Nonlinear Partial Differential Equations, Lecture Notes in Mathematics 1627, pages 48-196. Springer, 1996.

[20] D. Talay. Discretization of stochastic differential equations. application to simulation. stochastic numerical methods for partial differential equations. In P. Neittaanmaki, T. Tiihonen, and P. Tarvainen, editors, ENUMATH 99 - Proceedings of the 3rd European Conference on Numerical Mathematics and Advanced Applications. World Scientific, Singapore, 2000. 